Michael ALVAREZ-PEREYRE

Université Paris-Sorbonne (Paris IV) - CELTA

\title{
L'impératif en anglais : «Préfiguration » D'UNe SORTIE du Système DE LA PAROLE
}

\section{Introduction* $^{*}$}

Un apport majeur du Cercle de Prague est l'idée que l'asymétrie centrepériphérie est l'un des paramètres constitutifs des systèmes linguistiques (DANEŠ, 1966; VACHEK, 1966). Étudier un système consiste alors à déterminer non seulement les relations qui le caractérisent de manière pertinente, mais aussi les degrés d'adhésion à ces relations. VACHEK (ibid. : 33-34) est allé plus loin en émettant l'hypothèse que, outre la tendance reconnue générale des systèmes à l'intégration de leurs éléments, il pourrait exister une tendance «anti-intégrative ». Cette hypothèse est fondamentale car elle suggère que la position périphérique de certains éléments ne serait pas forcément le simple résultat de changements connexes (approche que Vachek attribue à Jakobson) mais qu'elle pourrait avoir une motivation fonctionnelle. Les exemples proposés par Vachek mettent en jeu la fonction émotive - émergeant à la marge de ce qui est pertinent d'un point de vue communicatif —, mais on peut penser, d'une part, que l'hypothèse reste valable pour d'autres fonctions du langage et, d'autre part, que la distinction entre centre et périphérie pourrait parfois tenir moins aux fonctions remplies qu'aux moyens de le faire. À la lumière d'une lecture de la construction du sens de l'impératif anglais, les paragraphes qui suivent appuient et développent ainsi l'hypothèse de Vachek dans le domaine conatif, en proposant que le positionnement de certains éléments vers le centre ou la périphérie d'un système linguistique pourrait refléter l'exploitation d'une compréhension globale et métalinguistique du système à des fins communicatives.

Dans la définition morphosyntaxique proposée par DAVIES (1986: §1.2) et acceptée par le plus grand nombre aujourd'hui, l'impératif anglais se caractérise (a) par l'absence possible et même typique de sujet, (b) par l'emploi de la base nue du verbe ${ }^{1}$ et (c) par l'emploi systématique de Do pour l'emphase et la négation (y compris avec Be et Have). Ces critères isolent une classe dont la définition ne se réduit pas à celle de la forme verbale (en anglais contemporain, le verbe à l'impératif n'est morphologiquement pas distinct de l'infinitif ou du subjonctif présent) mais correspond en fait à un type d'énoncé, c'est-à-dire à une manière d'employer cette forme (cf. JESPERSEN, 1954: §10.1). C'est à la construction du sens de ces énoncés que l'on s'intéressera ici, en s'en tenant à l'emploi majoritaire des impératives simples instanciant des requêtes (ordres, invitations, prières, etc.). Une tradition bien implantée, héritée de la pragmatique

\footnotetext{
* Cette recherche a bénéficié du soutien financier de l'Université Paris-Sorbonne (contrat n 15955 2005) ainsi que du CELTA et de l'École Doctorale V, que je remercie vivement. Je remercie mon Directeur de recherches, Pierre Cotte, ainsi que Frank Alvarez-Pereyre, pour de nombreuses discussions sur les sujets abordés ici et pour leurs relectures; les participants au colloque et les membres du comité scientifique pour leurs questions et leurs commentaires.

${ }^{1}$ Ceci apparaît notamment avec Be (Be quiet) et dans les impératives dites « de troisième personne » (Somebody help me!).
} 
traditionnelle, considère les requêtes à l'impératif comme des actes de langage « directs »: la reconnaissance de l'impératif permet la perception immédiate d'une tentative d'influence, dont l'objectif est explicite (par ex. Close the window). Il n'y a donc pas besoin d'une construction du sens élaborée - contrairement à celle qui permet d'interpréter It is cold in here comme requête. On est en présence d'une convention qui joue son rôle.

Cette approche est probablement juste du point de vue de l'usage, et un fonctionnalisme descriptif peut s'en contenter: à un signifiant spécifique correspond par convention un sens donné. Un fait empirique remarquable encourage cependant à chercher un fondement à cette convention : un grand nombre de langues spécialisent la ou l'une des formes les plus courtes de leurs verbes pour instancier des tentatives d'influence (BIRJULIN \& XRAKOVSKIJ, 2001 : §; HASPELMATH et al., 2005: \$70). Dans le cadre d'une sémantique grammaticale explorant la mise en forme du sens (GuILLAUME 1929: 1-6; BENVENISTE, 1966 : chap. IV ; COTTE, 1998) on peut se demander si l'usage de l'impératif anglais n'est pas sous-tendu par une construction du sens suffisamment conventionnalisée pour n'être pas rejouée à plein à chaque emploi de l'impératif, mais permettant néanmoins d'en comprendre la morphosyntaxe spécifique. Ceci revient, de fait, à tenter d'expliquer en partie comment et pourquoi la brièveté peut être exploitée par la langue à des fins directives, constat souvent fait - exposé clairement par exemple chez JESPERSEN (1922 : XX.\$9) ou plus récemment chez GIVON (1993 : 265-266) — mais rarement argumenté. Tout le monde semble s'accorder à dire que le «direct » est plus «directif »; mais au juste en vertu de quoi l'est-il ?

C'est pour l'anglais seul qu'une réponse sera proposée ici. La raison en est que le sens n'offre de prise qu'au sein d'une langue donnée, et que la «brièveté » est une notion systémique : un énoncé, impératif ou autre, n'est pas plus ou moins «direct» aux oreilles de qui ne comprend pas la langue; et Close the window («Ferme la f(e)nêtre ») n'est, en au moins un sens, pas plus «bref » que I'm cold («J'ai froid »). ${ }^{2}$ La motivation recherchée ici dans l'exploitation d'une certaine «brièveté » sera donc, par nécessité, «endo-systématique » (BOONE \& JOLY, 2004 : article «Arbitraire»), c'est-à-dire «se référant à la logique interne du système de la langue ». Le fonctionnement de l'impératif anglais sera ainsi relié à la position que sa morphosyntaxe lui fait prendre à la périphérie d'un système, celui de la « syntagmatisation ${ }^{3}{ }^{3}$ Rien ne sera cependant préjugé d'autres langues,

\footnotetext{
${ }^{2}$ L'intonation peut éventuellement aider l'auditeur étranger à détecter une certaine «expressivité », mais attribuer à celle-ci une valeur exige normalement la connaissance du système linguisticoculturel d'énonciation. Au sein même d'un tel système, il faut de plus souligner que, contrairement à une idée courante retrouvée jusque chez un BenvenISTE (1966: 274-275), l'impératif n'est pas énoncé avec une "intonation spécifique». Divers contours intonatifs se rencontrent, contribuant, comme le note déjà WEBSTER (1833 [1831] : 62), à la diversité des actes illocutoires que l'impératif peut réaliser (suggestions, ordres, prières, etc.); et il a été proposé, pour l'anglais, que ces intonations seraient moins spécifiques à l'impératif qu'aux différents actes illocutoires instanciés, qui peuvent l'être avec les mêmes intonations portant sur d'autres structures (BOLINGER, 1982; 1989: 150 sq.). Qui ignore la morphosyntaxe d'une langue ne peut donc pas a priori reconnaitre un impératif, que ce soit à la longueur de l'énonciation ou à son intonation.

${ }^{3}$ Il est fondamental de noter, à la suite de DANEŠ (1966:13), que l'« intégration » d'un élément au sein d'un système peut être évaluée à différents niveaux, notamment selon que l'on observe : (a) le
} 
L'impératif en anglais : «préfiguration » d'une sortie du système de la parole

lesquelles, dotées d'un système verbal différent, d'un fonctionnement morphosyntaxique différent, etc., pourraient instancier, ou non, cette tendance, de manière similaire ou distincte.

\section{Le cadre théorique : approcher l'impératif par le sens}

\subsection{Davies et la « présentation d'une potentialité de procès »}

Dans son ouvrage incontournable sur l'impératif anglais, DAVIES (1986: §2.3) observe ce qui se donne au co-énonciateur dans le signifiant d'une proposition à l'impératif: un radical sans marques de mode, de temps ou de personne (y compris pronom). La présence et la position de telles marques diraient quelque chose de l'actualisation du procès, plaçant celui-ci sur une certaine carte du monde (passé, présent, futur, réel, irréel, lié à une personne, etc.). Par contraste, la base nue du verbe renvoie seulement à un procès vu sous l'angle de sa «potentialité » la plus indéterminée ${ }^{4}$; or, à l'impératif, cette base n'est pas régie par un verbe placé en amont dans le linéaire mais seulement «présentée » dans la parole. Le fonctionnement de l'impératif anglais repose ainsi, dit Davies, sur la présentation, par l'énonciateur au co-énonciateur, d'une potentialité de procès. Qu'il faille mettre cette potentialité en acte n'est cependant pas signifié, pour Davies, par la structure elle-même, mais impliqué par une «convention d'usage » pragmatique qui en accompagne l'énonciation: de même, dit-elle, qu'une personne énonçant une déclarative est supposée dire la vérité, de même une personne énonçant une impérative est-elle supposée accepter l'actualisation du procès dont elle présente la potentialité. Puis, le co-énonciateur interprète cette acceptation en fonction du contexte et comprend l'énoncé comme ordre, suggestion, etc. On peut cependant se demander si dans la grammaire elle-même de l'impératif, il n'y aurait pas des éléments qui déjà anticipent et favorisent le passage à l'acte. C'est la question que s'est posé COTTE (1998 : chap. 13).

\subsection{Cotte et la «préfiguration mimétique »}

Pour comprendre l'intérêt de la démarche cottienne, revenons sur le statut qui a pu être donné à l'absence de marques de l'impératif anglais. Chez les premiers générativistes, cette absence était vue "négativement», comme un manque à expliquer en postulant des règles d'effacement à partir d'une structure déclarative sous-jacente (KATZ \& POSTAL, 1964 ; POSTAL, 1964 ; etc.). Une approche que l'on peut qualifier de «neutre» est celle, purement conventionnaliste, selon laquelle il suffit que l'impératif se distingue des autres formes verbales (en

positionnement structurel par rapport aux autres éléments du système, (b) le rendement fonctionnel de l'élément ou (c) la fréquence d'usage en nombre d'occurrences dans la parole. Il n'est pas rare que ces critères divergent. Dans le cas présent, M.-C. Pouder fit remarquer lors du colloque à quel point l'impératif occupe une place «centrale » durant la période d'acquisition de la parole chez les enfants. La pragmatique a par ailleurs attribué une place centrale aux énoncés impératifs en y voyant la norme directe des requêtes, par rapport à laquelle se définissent les stratégies dites « indirectes ». La position périphérique dont je traite ici est à entendre sur un plan morphosyntaxique : sens (a).

4 Benveniste (1966 : 274-275) reconnaît déjà dans l'impératif le « sémantème nu »; BoLINGER (1977 : 196-197) et DownES (1977 : 86), qui voient dans l'impératif un emploi particulier de l'infinitif, parlent déjà d'action « hypothétique »; mais l'analyse de la structure dans son ensemble revient à Davies. 
l'occurrence, par la marque zéro) pour qu'on le reconnaisse, sans que ces moyens formels n'aient nécessairement de lien avec le sens. Ces moyens sont parfois justifiés dans une argumentation de type pragmatique attestée depuis longtemps, ${ }^{5}$ convaincante chez DOWNES (1977 : 83) mais le plus souvent rencontrée dans la version intuitive suivante $:^{6}$ l'agent souhaité étant présent devant l'énonciateur et l'actualisation étant souvent censée être immédiate, la mention d'un sujet ou de marques de temps n'est pas nécessaire. Cette idée séduisante (dans laquelle l'absence de marque est encore vue de manière «neutre », suivant l'idée qu' «il n'est pas nécessaire de marquer ce qui est évident en situation ») n'a, à vrai dire, rien d'assuré : il pourrait être en fait plus coûteux pour une langue de conserver une partie de sa grammaire du verbe sans sujet (et ce, de manière systématique, quel que soit le registre) alors que la majorité des structures en prennent un (y compris celles du type It is raining, etc.). Si une certaine «évidence» situationnelle que l'auditeur est l'agent projeté peut éventuellement autoriser la non-mention d'un sujet, elle ne suffit donc peut-être pas à expliquer que cette omission devienne norme. Dans une perspective de motivation, l'intérêt de Davies est de donner un statut « positif » à l'absence de marques, en voyant cette absence comme signifiante puisqu'elle permet de présenter le procès sous l'angle de sa potentialité. L'idée de Cotte est d'aller plus loin, en voyant dans l'absence de marques le ressort non seulement du sens mais aussi de l'efficacité de l'impératif.

Pour Cotte, cette efficacité tient à la manière «implicite » dont l'impératif communique la modalité. Expliciter celle-ci (par exemple avec You should...) ferait saillir des éléments symbolisant un possible retard dans la délibération du co-énonciateur («pourquoi moi ?», «pourquoi devrais-je ?»). La référence exclusive au procès visé permet ainsi de «détourner l'allocutaire de toute délibération pour qu'il relaie sans tarder la visée exprimée et qu'il quitte le monde des mots pour celui des actes » (COTTE, 1998: 126-127). Cette référence exclusive permet, de plus, d'opérer une «préfiguration mimétique »(ibid.) : le minimalisme de l'énoncé (qui magnifie l' «injonction permanente» de tout discours, celle d'écouter) met en avant l'énonciation elle-même. Le défaut de parole laisse ainsi transparaître l'énonciateur dans son acte d'énonciation, c'est-àdire comme acteur. En se manifestant dans ce rôle, l'énonciateur montre le chemin, dont le contenu énoncé donne la direction. Cotte propose ainsi de voir dans les signes énoncés et dans la manière dont ils sont énoncés un renvoi vers le monde de l'agir. Dans la lignée de cette hypothèse, on peut proposer d'approfondir l'étude de la «préfiguration mimétique » qu'opère l'impératif, en observant un refus général de jouer le jeu de la parole.

\section{L'impératif comme «préfiguration » d'une sortie du système de la parole}

L'hypothèse proposée ici porte l'idée de «potentialité »sur un autre plan que celui considéré jusqu'à présent. Avec Davies, on s'est concentrés sur la base nue $\mathrm{du}$ verbe en tant qu'elle permet de présenter un contenu conceptuel (en l'occurrence une action) sous l'angle de sa potentialité. À cette analyse, on peut ajouter une dimension supplémentaire : car la base nue du verbe, présentée au

\footnotetext{
${ }^{5}$ Cf. Kellner (1892: \$452.1).

${ }^{6}$ Cf. par exemple Givon (1993 : 266).
} 
destinataire sans marques de personne (pronom y compris), de mode ou de temps, est finalement la version la plus brute du verbe, celle qui se rapproche le plus de son identité en langue. La «potentialité » sur laquelle repose la construction du sens de l'impératif pourrait donc être également, et fondamentalement, linguistique.

Que l'impératif soit perçu comme plus «direct» serait ainsi à relier à un «effet de potentialité linguistique»: tout se passe comme si l'énonciateur saisissait le verbe brut et le plaçait dans le syntagmatique, en faisant l'économie des processus de linéarisation qui scellent habituellement l'entrée dans le monde de la parole. Ceci peut faire sens car, par opposition à la langue mais aussi au monde extralinguistique, la parole est, fondamentalement, le monde où sont posées et négociées les relations entre des concepts, des représentations, des éléments indexicaux, etc. Un signe certain que l'on est dans le monde de la parole est donc le système des marques qui disent la relation entre un procès et de la personne, de la temporalité, de la realis, de la modalité, etc. Or, la particularité de l'impératif anglais est de faire l'économie systématique de ces marques. Pourtant, on ne peut nier l'existence d'opérations cognitives mettant en relation un procès, une personne, une temporalité, etc. : l'énonciation de toute requête suppose que de telles opérations mentales aient eu lieu. Simplement, ces relations ne sont pas enregistrées, elles ne sont pas signalées à l'impératif (alors qu'elles le sont encore en partie au subjonctif et à l'infinitif, cf. §3). Peut-être y a-t-il ici du «faire semblant », c'est-à-dire simplement un « masquage » des opérations d'entrée dans la parole, dont la trace est précisément dans les marques mentionnées. La raison pourrait en être qu'en s'abstenant d'utiliser les marques de linéarisation (de « syntagmatisation ») qui sont le signe même de la parole actualisée, l'impératif anglais serait mieux à même de «préfigurer » une sortie du système de la parole, en participant de manière minimale aux relations qui caractérisent ce système, pour employer les termes de VACHEK (1966 : 23). «De manière minimale », dans une mesure à préciser, car il n'y a pas absence totale de linéarisation : l'ordre des constituants verbalisés reste contraint, et les syntagmes à droite du verbe sont construits régulièrement. L'impératif n'est donc pas « hors-système » mais plutôt à observer à la périphérie d'un système, celui de la linéarisation prédicative. ${ }^{7}$ Il existe des structures moins linéarisées, susceptibles d'instancier des requêtes que d'aucuns jugeraient plus «directes » : syntagmes prépositionnels (Over there!) et nominaux (The door!), ou encore interjections (Hey!, Lo!). L'impérative est cependant la structure à verbe la plus périphérique en anglais, dans le système de la linéarisation. ${ }^{8}$ Cette position est exploitable fonctionnellement, car ce qui peut être perçu comme le refus - réel ou simulé - d'une étape de construction linguistique (c'est à dire d'une partie du temps de la linéarisation) peut servir à signifier l'urgence. Cette idée repose, en définitive, sur un rapprochement entre les

\footnotetext{
${ }^{7}$ On peut noter que Cercle de Prague a très tôt considéré la prédication comme «l'acte syntagmatique fondamental », comme l'a montré RAYNAUD (2008).

${ }^{8}$ Les déclaratives, interrogatives, exclamatives à verbes et subordonnées portent à divers degrés des marques de personne, de temps, de mode, de modalité et de relations propositionnelles. L'analyse des types de marques et de leur positionnement permettrait de complexifier les rapports au linéaire, mais il suffit ici de signaler une opposition générale avec les impératives.
} 
notions d'antériorité et d'extériorité, dont le rapport, bien connu dans les langues, ${ }^{9}$ est fortement ancré en anglais — en atteste l'emploi du prétérit pour coder le passé aussi bien que l'irréel (ces deux plans ont en commun de ne pas être actuels au moment de l'énonciation). Peut-être est-ce cette affinité qui joue à l'impératif, avec l'exploitation d'une posture pré-linguistique pour mieux signifier une visée extralinguistique : car le refus, réel ou simulé, d'entrer pleinement dans le monde de la parole est peut-être le meilleur moyen d'en sortir. L'incitation à « quitte[r] le monde des mots (...) » (COTTE, ibid.) ne résiderait alors pas seulement dans le fait de ne pas expliciter ce qui pourrait arrêter l'attention, mais aussi dans un effet de rupture avec la trame de l'échange verbal, dans le «mimétisme » d'un débrayage de la parole vue en tant qu'elle est un certain rapport au monde et à l'action (un rapport d'analyse et d'articulation plutôt que de contact) — rapport qu'il faut emprunter pour requérir de vive voix mais qui, à l'impératif, est déjà nié dans la manière de requérir.

\section{L'impératif comme « retour » vers la situation}

Que ce soit à une sortie de la parole que le co-énonciateur est invité est appuyé par une convergence entre le fonctionnement énonciatif avancé ci-dessus et le profil sémantico-référentiel des propositions à l'impératif. Les résultats préliminaires d'une étude statistique en cours ${ }^{10}$ révèlent que, contrairement à une idée reçue, ${ }^{11}$ l'énoncé impératif prototypique ne se réduit pas au verbe seul. Dans l'échantillon étudié, environ un verbe à l'impératif sur deux régit un complément d'objet, et parfois deux. Or, ces objets suivent une tendance forte (72\%) à la définitude situationnelle (Look at this, Listen to me, Close the door, etc.).

Dans les propositions impératives sans complément d'objet, on observe une multitude de compléments circonstanciels obligatoires qui jouent un rôle définitoire vis-à-vis du procès, le plus souvent en nommant le site où l'action doit se réaliser ou s'achever (Sit over there, Get out of the pool, etc.). De très nombreux verbes dits «à particule» suivent un même schéma (Sit down, Come in, etc.). Les verbes sans compléments d'objet ont ainsi une forte tendance $(70 \%)$ à être suivis d'un élément non supprimable défini sémantiquement par renvoi à la situation.

Ces tendances (ainsi que d'autres, plus complexes, non discutées ici) révèlent que les énoncés à l'impératif «pointent» vers la situation, en vertu d'une complémentation à droite riche, majoritairement définie, et ce de manière situationnelle. Dans le linéaire, l'orientation du regard vers cette complémentation est renforcée, d'une part, par le dynamisme des procès (essentiellement des actions transitives ou des déplacements, dont la valence suscite une recherche de compléments à droite) et, d'autre part, par leur simplicité sémantique (qui accroît

\footnotetext{
${ }^{9}$ Cf. par exemple les communications de MM. KISS et ACHARD-BAYLE, ce volume.

${ }^{10}$ L'étude porte sur un corpus de films nord-américains contemporains. L'échantillon étudié, composé de 594 propositions impératives simples, correspond à un échantillon initial d'environ 700 impératives dont ont été exclues les périphrases en Let/Make et les structures aspectuelles du type $\langle$ Sart/Keep/... + V-ing $>$.

11 Rencontrée encore récemment dans l'introduction d'un ouvrage consacré intégralement à l'impératif (WURFF, $2007: 84$ ).
} 
l'importance informationnelle des compléments). ${ }^{12}$ La forme lexicale en ellemême est remarquable, puisque $90 \%$ des verbes choisis par les locuteurs sont monosyllabiques $^{13}$ (une fois les verbes à particules comptés comme un verbe suivi d'un complément, cf. supra). S'ajoutant à l'absence typique de sujet, ce minimalisme généralisé (sémantique autant que formel) du verbe à l'impératif, permet une focalisation forte de la fin de l'énoncé. Or, celle-ci est typiquement constituée de compléments dont la définitude situationnelle souligne une préexistence et une transcendance vis-à-vis du discours. On peut penser que cette structuration tendancielle des énoncés impératifs renforce et précise l'incitation, décrite plus haut, à sortir du monde de la parole, cette sortie étant présentée sur le mode d'un retour vers la situation à modifier.

\section{L'exploitation d'une possibilité de motivation}

Il est temps de préciser les conditions dans lesquelles a pu s'exercer ce que VACHEK (1966 : 33) appelle la tendance «anti-intégrative ». Le terme n'évoque pas le mouvement vers la périphérie mais bien le frein à l'intégration vers le centre. De fait, il ne faudrait pas croire que la morphosyntaxe de l'impératif anglais serait le résultat direct et attendu d'un mouvement téléologique qui aurait fait perdre à ce mode les marques - réduites mais existantes (cf. MILLWARD, 1971) —, qu'il avait en vieil anglais, ceci pour se retrouver à la périphérie d'un système, etc. Il serait d'ailleurs sans doute abusif de croire que la perte des marques de l'impératif aurait des «causes » bien différentes de celles qui ont mené le verbe anglais (et l'anglais de manière globale) sur la voie d'une réduction morphologique très généralisée. Ce qui est spécifique à l'impératif, en revanche, c'est la manière dont cette réduction a été «monnayée », comme le montre la comparaison avec l'infinitif et le subjonctif.

L'infinitif en vieil anglais était marqué d'un suffixe spécifique (-an), et les structures qui l'introduisaient n'employaient pas l'élément to. En anglais moderne, le suffixe a disparu et l'infinitif est souvent précédé de to, au point que ce sont les rares structures introduisant directement la base verbale qui semblent devoir mériter explication. VALLINS (1957 : 17) présente la systématisation de l'emploi de to avec l'infinitif comme une «compensation» de la perte flexionnelle, éclairant ainsi l'évolution de ce mode sous l'angle de son intégration dans le linéaire - désormais assurée de manière syntaxique et non plus morphologique. ${ }^{14}$ Au subjonctif, qui s'est également vu réduire à la base nue du verbe, il n'y a pas eu de développement comparable à l'émergence de to. On note cependant une

\footnotetext{
${ }^{12}$ Dans le corpus étudié, les vingt verbes les plus fréquents (qui fournissent les deux tiers des énoncés impératifs) sont, par ordre décroissant d'emploi : Come, Get, Look, Hold, Tell, Listen, Take, Go, Wait, Give, Excuse, Watch, Sit, Be, Say, Worry, Check, Bring, Shut, Put.

${ }^{13}$ Pour COTTE (2008: $\$ 4.3$; communications personnelles) « notre rapport à l'action varie selon que nous agissons ou que nous analysons l'action ; le monomorphémisme exclut l'attitude analytique. Il s'agit d'un mimétisme, d'une transposition dans l'ordre des signes d'une attitude en dehors des signes ».

${ }^{14}$ Cette «compensation» va jusqu'à prendre une dimension paradigmatique dans l'idée (abusive cependant) que to ferait partie de l'infinitif. Cette idée est présente en creux dans l'expression d' « infinitif sans to » souvent rencontrée dans les grammaires (SIMON, 1994 ; LARREYA \& RIVIERE, 2002 ; etc.).
} 
marginalisation de l'emploi de ce mode, dans la conversation mais également à l'écrit (son usage est parfois même déconseillé aux apprenants de l'anglais). On rencontre désormais, pour l'essentiel, soit un indicatif (Take your umbrella in case it rains), soit une périphrase modale (...in case it should rain; They are afraid he might run away, etc.). Que ce remplacement du subjonctif morphologique par d'autres tournures, notamment à auxiliaires modaux (ROMAINE, 1998: 160), puisse avoir pour origine un sentiment de «manque » d'intégration est suggéré par une remarque de KELLNER (1892 : 234), selon lequel les auxiliaires modaux sont employés «pour compenser la perte des formes perceptibles du mode subjonctif ${ }^{15}$ En tout état de cause, ce remplacement, qui permet l'explicitation de la modalité, est cohérent dans la perspective d'une évolution analytique de l'anglais.

L'impératif, alors même qu'il présente la morphologie la plus réduite possible, prend sa place dans le linéaire sans «compensation » syntaxique comparable à celles développées par l'infinitif ou le subjonctif. L'évolution de ces deux modes montre pourtant, s'il le fallait, la capacité de l'anglais à développer des stratégies pour expliciter la modalité et assurer l'inscription de la base nue du verbe dans le syntagmatique. L'histoire de l'impératif peut apparaître alors, par différenciation, comme la sélection (ou l'accentuation) d'un rapport au linéaire radicalement différent des autres modes, rapport caractérisé par un « minimalisme d'intégration » qui correspond historiquement à une réduction (ou plus récemment à un défaut) morphologique non compensée par un développement syntaxique. Vue sous cet angle, l'histoire de l'impératif anglais serait l'histoire de la préservation d'une position périphérique dans le système de la linéarisation, parce que cette position pouvait être exploitée à des fins communicatives (cf. §1 et §2).

\section{Conclusion}

L'étude a proposé un exemple concret, dans le domaine de la grammaire du verbe, de la manière dont la tendance «anti-intégrative » supposée par VACHEK (1966) peut être liée à l'exploitation de possibilités fonctionnelles particulières. Pour le cas étudié, l'accent peut être mis sur deux points: premièrement, la position périphérique est préservée et exploitée (et non pas «recherchée » de manière finaliste), ce qui appuie le choix du terme «anti-intégration » plutôt que «périphérisation». Deuxièmement, le potentiel fonctionnel lié la position périphérique ne correspond pas à une fonction proprement différente de celle prise en charge au centre du système (cf.l'opposition fonction émotive vs. communicative dans l'article de Vachek) mais semble tenir plus généralement aux moyens de communiquer du sens, avec l'exploitation d'une compréhension métalinguistique de ces moyens. ${ }^{16}$

\footnotetext{
${ }^{15}$ Le sentiment de «manque » d'intégration ressenti dans l'emploi du subjonctif «morphologique » a pu pousser certains linguistes à voir cette forme - explicitement (PRIESTLEY, 1833 [1761] : $\mathrm{V}_{\text {II }}$; COBBETT, 1817 : §95) ou implicitement (SIMON, 1994: §117) — comme une périphrase modale (notamment en should) faisant l' «ellipse » de l'auxiliaire.

${ }^{16}$ L'opposition de Vachek pourrait cependant être relue plus abstraitement comme l'hypothèse d'une affinité entre périphérie et expressivité.
} 
La lecture proposée de la construction du sens de l'impératif anglais doit encore être confrontée avec la diversité illocutoire ${ }^{17}$ et structurelle $^{18}$ des impératives. Elle espère néanmoins avoir contribué à l'étude de l'instanciation, dans la logique interne d'une langue donnée, de la tendance supposée assez générale à l'exploitation du «bref » pour réaliser des fonctions d'incitation à l'action.

\section{BIBLIOGRAPHIE}

BenVEniste, Émile (1966), Problèmes de linguistique générale, vol. I, Paris, Gallimard.

BIRJUlin, Leonid A., XRAKOVSKIJ, Viktor S. (2001), Imperative sentences: Theoretical problems, in : XRAKOVSKIJ Viktor S. (éd.), Typology of imperative constructions (LINCOM Studies in Theoretical Linguistics 09), Munich, Lincom Europa, p. 3-50.

BOLINGER, Dwight L. (1977), Meaning and form, Londres, Longman.

BolingER, Dwight L. (1982), Nondeclaratives from an intonational standpoint, in : SCHNEIDER, Robinson, TuITE, Kevin, CHAMETZKY, Robert (éds.), Papers from the parasession on nondeclaratives, Chicago, CLS.

BOLINGER, Dwight L. (1989), Intonation and its uses, Stanford, Stanford University Press.

Boone, Annie, Joly, André (2004), Dictionnaire terminologique de la systématique du langage [2 ${ }^{\text {nde }}$ édition], Paris, L'Harmattan.

COBBETT, William (1919 [1817]), A grammar of the English language, revised and annotated by Alfred Ayres, Londres, D. Appleton and Company.

CoTTE, Pierre (1998 [1996]), L'Explication grammaticale de textes anglais, Paris, Presses Universitaires de France.

CotTe, Pierre (2008), Expressivité secrète, Bulletin de la Société de Stylistique Anglaise 30, p. 11-27, [http://stylistique-anglaise.org/document.php?id=508] (Consulté le 10 mai 2009).

DANEŠ, František (1966), The relation of centre and periphery as a language universal, Travaux linguistiques de Prague 2 : Les problèmes du centre et de la périphérie du système de la langue, Prague, Academia, p. 9-21.

DAVIES, Eirlys (1986), The English imperative, Beckenham, Croom Helm.

DOWNES, William (1977), The imperative and pragmatics, Journal of Linguistics, 13, p. 77-97.

GIVÓN, Talmy (1993), English grammar: a function-based introduction, vol. II, Amsterdam, John Benjamins.

Guillaume, Gustave (1929), Temps et verbe : théorie des aspects, des modes et des temps, Paris, H. Champion.

\footnotetext{
${ }^{17}$ Certains emplois de l'impératif (défis, suggestions, modes d'emploi) ne supposent pas que l'énonciateur désire un passage à l'acte. La «préfiguration » d'une sortie de la parole est-elle alors exploitée sur un mode métaphorique ? Ou faut-il, à la suite de DAVIES (1986), prendre une position de repli et placer le ressort de l'incitation à l'action hors la grammaire de l'impératif ?

18 Impératives en Let, impératives négatives ou emphatiques, impératives avec expression de personne, impératives en protase de conditionnelle, etc.
} 
HASPelmath, Martin, Dryer, Matthew S., GIL, David, ComRIE, Bernard (éds., 2005), The world atlas of language structures, Oxford, Oxford University Press.

JESPERSEN, Otto (1922), Language: its nature, development and origin. London, George Allen \& Unwin Ltd.

JESPERSEN, Otto (1954), A modern English grammar on historical principles, partie V («Syntax »), vol. IV, London, George Allen \& Unwin Ltd.

KatZ, Jerrold J., Postal, Paul M. (1964), An integrated theory of linguistic descriptions, Cambridge, MIT Press.

KELLNER, Leon (1892), Historical outlines of English syntax, London, Macmillan and Co.

LARreyA, Paul, RIVIERE, Claude (2002), Grammaire explicative de l'anglais, London, Pearson Longman.

Millward, Celia M. (1971), Imperative constructions in Old English (Janua Linguarum Series Practica 124), La Haye, Mouton.

Postal, Paul M. (1964), Underlying and superficial linguistic structure, Harvard Educational Review, 34, p. 246-266.

PRIESTLEY, Joseph (1833 [1761]), The rudiments of English grammar, dans English grammar; Lectures on the theory of language and universal grammar; and on oratory and criticism. London, Rowland Hunter.

RAYNAUD, Savina (2008), The basic syntagmatic act is predication, Slovo a slovesnost, 69, p. 49-66.

Romaine, Suzanne (éd., 1998), The Cambridge history of the English language, Vol. IV, Cambridge, Cambridge University Press.

SIMON, Pierre (1994), Grammaire anglaise: De l'exemple à la règle, Paris, Ellipses.

VACHEK, Josef (1966), On the integration of the peripheral elements into the system of language, Travaux linguistiques de Prague 2: Les problèmes du centre et de la périphérie du système de la langue, Prague, Academia, p. 2337.

VALLINS, George H. (1957 [1956]). The Pattern of English, Londres, Pelican.

WEBSTER, Noah (1833 [1831]). Improved grammar of the English language. New Haven, Durrie and Peck.

WURFF, Wim van der (éd., 2007), Imperative clauses in generative grammar: Studies in honour of Frits Beukema (Linguistik Aktuell 103), Amsterdam, John Benjamins. 


\section{SUMMARY}

This paper tackles aspects of the construction of meaning of the English imperative, in the framework of Vachek's (1966) suggestion that "antiintegrating" tendencies may be at work in linguistic systems when the peripheral position of some elements enables them to activate specific functional possibilities. The typical lack of markers in the imperative is claimed to contribute positively to a particular strategy of enunciation. Davies (1986) showed how the bare stem of the verb enables the speaker to present the hearer with a potentiality of action to be enacted. To account for the way the projection of actual enactment is signified, Cotte (1998) advanced the concept of "mimetic prefiguration" (the idea that the lack of markers casts light on the act of uttering itself, thus implicitly laying the focus on the idea of "action"). This paper proposes an addition: the verb base, which is presented to the addressee without any markers of tense, mode or person (including pronouns), is the version of the verb closest to its identity in "langue". I suggest that the systematic absence of syntagmatic markers positions imperative utterances at the periphery of the system of linearized speech, a position which enables these utterances to "prefigure" the desired shift from the world of talk to that of action. 